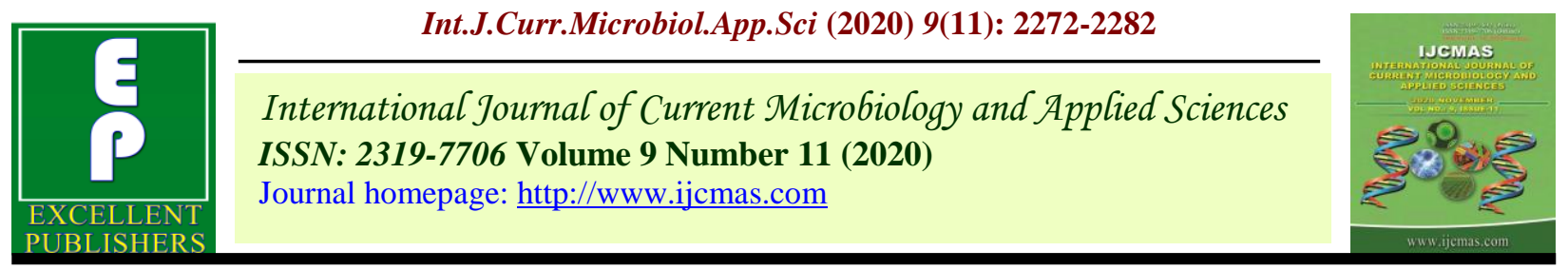

Original Research Article

https://doi.org/10.20546/ijcmas.2020.911.272

\title{
Effect of Mulching, Drip irrigation and Fertigation on Growth, Flowering and Yield parameters of Nerium (Nerium oleander L.)
}

\author{
K. Annasamy ${ }^{1^{*}}$, S. Muthu Lakshmi ${ }^{1}$, K. M. Sellamuthu ${ }^{2}$, T. Thangaselvabai ${ }^{3}$, \\ J. Kannan ${ }^{2}$, T. L. Preethi ${ }^{1}$ and P. Arularasu ${ }^{1}$ \\ ${ }^{1}$ Department of Floriculture and Landscape Architecture, ${ }^{2}$ Department of Natural Resource \\ Management, Horticultural College and Research Institute, Peroyakulam, India \\ ${ }^{3}$ Horticultural Research Station, Thadiankudisai, India \\ *Corresponding author
}

\section{A B S T R A C T}

Keywords

Mulching,

Drip irrigation,

Fertigation,

Growth, Yield,

Quality, Nerium

Article Info

Accepted:

17 October 2020

Available Online:

10 November 2020
A field experiment was conducted to study the effect of mulching, drip irrigation and fertigation on growth, yield and quality of nerium (Nerium oleander L.) at Department of Floriculture and Landscape Architecture, Horticultural College and Research Institute, Tamil Nadu Agricultural University, Periyakulam during 2016-2020. The experiment was laid out in a split split plot design consisting of three factors, viz., factor - I $\left(\mathrm{M}_{1}-\mathrm{Black}\right.$ polythene mulch, $\mathrm{M}_{2}$-coir waste and $\mathrm{M}_{3}$-without mulch, factor - II $\left(\mathrm{I}_{1}-75 \% \mathrm{WRc}\right.$ through drip irrigation, $\mathrm{I}_{2}-100 \% \mathrm{WRc}$ through drip irrigation and $\mathrm{I}_{3}-125 \% \mathrm{WRc}$ through drip irrigation) and factor - III ( $\mathrm{F}_{1}-75 \% \mathrm{RDF}$ through fertigation, $\mathrm{F}_{2}-100 \% \mathrm{RDF}$ through fertigation, $\mathrm{F}_{3}-125 \%$ RDF through fertigation)with twenty seven treatment combinations. The results of the study indicated that $\mathrm{M}_{1} \mathrm{I}_{2} \mathrm{~F}_{3}$ (black polythene mulch $+100 \% \mathrm{WRc}$ through drip irrigation $+125 \%$ RDF through fertigation) recorded the highest plant height, number of primary branches, days taken to first inflorescence emergence and days to first flower opening and flower yield $(\mathrm{kg}) \mathrm{ha}^{-1}$.

\section{Introduction}

Nerium (Nerium oleander L) is an evergreen shrub belonging to Apocynaceae family and is native to North Africa. It is widely planted as an ornamental plant in warm temperate, tropical and sub tropical regions, survives drought well and is well suited for cultivation in even poor soils. This crop can tolerate salt sprays, brackish water and alkaline soil. It is commonly planted in highway medians as a no - maintenance plant. Oleander also does well as a quick growing screen or large specimen planting. Nerium flowers are commonly used for worship in home and temples and so is an important loose flower. In the present scenario of acute water shortage and unpredictable climatic conditions, effective and economic utilization of resources is essential and can be achieved through the use of improved techniques, viz., drip irrigation, fertigation and mulching. Drip irrigation results in discrete and efficient water usage. The moderation of soil 
hydrothermal regimes by application of mulching results in better nutrient uptake, water absorption, metabolite production and carbohydrate storage and is reflected in better growth and higher yield of crops. In addition, mulches control weed incidence, reduce nutrient losses and affect various physical, chemical and biological reactions involved in plant growth and development, besides considerable saving irrigation water. Fertigation ensures better quality water soluble fertilizers for commercial flower production in various situations (Muthu Kumar, 2013). Hence, mulching, drip irrigation and fertigation may prevent crop water stress and have considerable effect on growth, flower yield and quality of nerium. Therefore the present studies were undertaken to establish the effect of various mulches, drip irrigation and fertigation on nerium.

\section{Materials and Methods}

The experiment was conducted at Department of Floriculture and Landscape Architecture, Horticultural College and Research Institute, Tamil Nadu Agricultural University, Periyakulam in 2016-2020.The experimental field is situated at $77 \mathrm{E}$ longitude, 10 latitude and at an altitude of $300 \mathrm{~m}$ above mean sea level (MSL). The treatments were randomly allocated in split split plot design and replicated three times. The experiment consisted of three factors, viz., factor - I ( $\mathrm{M}_{1-}$ Black polythene mulch, $\mathrm{M}_{2}$-coir waste and $\mathrm{M}_{3}$-without mulch, factor - II ( $\mathrm{I}_{1}-75 \% \mathrm{WRc}$ through drip irrigation, $\mathrm{I}_{2}-100 \%$ WRc through drip irrigation and $\mathrm{I}_{3}-125 \% \mathrm{WRc}$ through drip irrigation) and factor - III ( $\mathrm{F}_{1}-75$ $\%$ RDF through fertigation, $\mathrm{F}_{2}-100 \%$ RDF through fertigation, $\mathrm{F}_{3}-125 \% \mathrm{RDF}$ through fertigation) with twenty seven treatment combinations. Four plants were selected randomly and data collected was subjected to statistical analysis as suggested by Panse and Sukhatme, 1985.

\section{Results and Discussion}

\section{Effect of mulching drip irrigation and fertigation on growth characters}

The results of the study on the effect of mulching, drip irrigation and fertigation on growth, yield and quality of nerium indicated that the individual as well as interaction effect had significant influence on morphological, flowering and yield characters. Among the three main plot treatments $\mathrm{M}_{1}$ (Black polythene mulch) recorded the highest plant height (151.0 and $216.5 \mathrm{~cm}$ respectively) and number of primary branches plant ${ }^{-1}$ in pre flowering and flowering stages (6.02 and 8.28 respectively). Among the three sub plot treatmentsI $I_{2} \quad(100 \%$ WRc through drip irrigation) recorded the highest plant height of (144.0 and $222.0 \mathrm{~cm}$ respectively) and number of primary branches plant ${ }^{-1}$ (5.67 and 7.31respectively) at pre flowering and flowering stages (Table $1 \mathrm{a}, 1 \mathrm{~b}$ and $2 \mathrm{a}$ and 2 b).

Among the three sub plot treatments, highest plant height was recorded duringpre flowering and flowering stagesin $\mathrm{F}_{3} \quad(125 \%$ RDF through fertigation) (152.5 and $196.3 \mathrm{~cm}$ respectively). Similarly highest number of primary branches plant $^{-1}$ (5.99 and 7.39 respectively) was also recorded in this treatment.The interaction effect between mulching and drip irrigation significantly influencedplant height. The treatment combination $\mathrm{M}_{1} \mathrm{I}_{2}$ (Black polythene mulch + $100 \%$ WRc through drip irrigation) recorded the highest plant height of (171.4 and $241.7 \mathrm{~cm})$ and number of primary branches plant $^{-1}$ (6.20 and 8.72) at pre flowering and flowering stages respectively.

Significant difference was observed in plant height due to the interaction effect of mulching and fertigation. The highest plant height of 195.6 and $242.9 \mathrm{~cm}$ and number of 
primary branches plant $^{-1}$ of 6.63 and 8.79 were recorded in $\mathrm{M}_{1} \mathrm{~F}_{3}$ (black polythene mulch $+125 \%$ RDF through fertigation) at pre flowering and flowering stages respectively. The combined effect of drip irrigation and fertigation on plant height was highly significant. The result showed that $\mathrm{I}_{2} \mathrm{~F}_{3}$ (100\% WRc through drip irrigation $+125 \%$ RDF through fertigation) exhibited the highest plant height of 182.9 and $271.7 \mathrm{~cm}$ and number of primary branches plant ${ }^{-1}$ (6.31 and 7.68) at pre flowering and flowering stages respectively.

The interaction effect of mulching, drip irrigation and fertigation indicated that the treatment $\mathrm{M}_{1} \mathrm{I}_{2} \mathrm{~F}_{3}$ (black polythene mulch + $100 \%$ WRc through drip irrigation $+125 \%$ RDF through fertigation) registered the highest plant height of 244.8 and $277.0 \mathrm{~cm}$ and highest number of primary branches plant $^{-1}$ (7.03 and 9.15) at pre flowering and flowering stages respectively and this was on par with $\mathrm{M}_{2} \mathrm{I}_{2} \mathrm{~F}_{3}$ (coir waste $+100 \%$ WRc through drip irrigation $+125 \%$ RDF through fertigation). This might be attributed to the fact that mulching resulted in favourable environmental conditions such as temperature, moisture and soil air, because of which the photosynthetic activity is increased. Application of drip irrigation and fertigation at frequent intervals provides a consistent moisture regime and nutrient pool in the soil and therefore, roots remain active for a longer period resulting in increased availability of nutrients to the plants and translocation of food materials which accelerate the vegetative growth of the plant besides maintaining the soil moisture as well as temperature at optimum level. This increases the yield as reported earlier by Chawla (2008) in african marigold, Yathindra (2009) in China aster; and Vijay Kumar (2012), Patel Bhaveshkumar Bharatbhai (2013), Vasanthakumari et al., (2013) and Alak Barman et al., (2015) in tuberose. Sufficient supply of nutrients at frequent intervals might have increased the production of IAA which consequently would have shown stimulatory action, in terms of cell elongation and thus resulting in increased plant height as mentioned by Jainag et al., (2011). This finding is in consonance with Vinoth (2012); Jakadeeshkanth (2014) and Khalid Elhindi et al., (2015).

Effect of mulching, drip irrigation and fertigation on days to first inflorescence emergence, days to first flower opening and yield characters of nerium

The results of the experiment indicated earliness in days to first inflorescence emergence and days to first flower opening under $\mathrm{M}_{1}$ - black polythene mulch (172.4) and (178.80) respectively (Table 3 and 4). Earliness was also observed in plants subjected to $100 \%$ and $75 \%$ WRc through drip irrigation $\left(I_{2}\right.$ and $\left.I_{1}\right)$. Similarly $125 \%$ RDF through fertigation $\left(\mathrm{F}_{3}\right)$ also recorded early inflorescence emergence and first flower opening (174.2 and 181.86 days respectively).

The interaction effects of mulching and drip irrigation significantly influenced days taken to first inflorescence emergence. The treatment $\mathrm{M}_{1} \mathrm{I}_{2}$ (Black polythene mulch +100 $\%$ WRc through drip irrigation) recorded early inflorescence emergence (172.1 and 178.02 days). The interaction effects of mulching and fertigation were significant with respect to days taken to first inflorescence emergence and days to first flower opening. The treatment $\mathrm{M}_{1} \mathrm{~F}_{3}$ (black polythene mulch + $125 \% \mathrm{RDF}$ through fertigation) recorded the least days to first inflorescence emergence (172.3 and 178.07 days) and higher days taken to first flowering opening.

The interaction effects of irrigation and fertigation were significant with respect to days taken to first inflorescence emergence. 
Table.1a Influence of mulching, drip irrigation and fertigation on plant height $(\mathrm{cm})$ in nerium at pre flowering stage

\begin{tabular}{|c|c|c|c|c|c|c|c|c|c|c|c|c|c|c|c|c|}
\hline Treatments & \multicolumn{4}{|c|}{$\mathbf{M}_{1}$} & \multicolumn{4}{|c|}{$\mathbf{M}_{2}$} & \multicolumn{4}{|c|}{$\mathbf{M}_{3}$} & \multicolumn{4}{|c|}{ I $x \mathbf{F}$} \\
\hline & $\mathbf{I}_{1}$ & $\mathbf{I}_{2}$ & $\mathbf{I}_{3}$ & Mean & $\mathbf{I}_{1}$ & $\mathbf{I}_{2}$ & $\mathbf{I}_{3}$ & Mean & $\mathbf{I}_{1}$ & $\mathbf{I}_{2}$ & $\mathbf{I}_{3}$ & Mean & $\mathbf{I}_{1}$ & $\mathbf{I}_{2}$ & $\mathbf{I}_{3}$ & Mean \\
\hline $\mathbf{F}_{1}$ & 101.1 & 122.3 & 127.3 & 116.9 & 96.8 & 127.9 & 121.8 & 115.5 & 70.5 & 89.1 & 88.7 & 82.8 & 89.5 & 113.1 & 112.6 & 105.1 \\
\hline $\mathbf{F}_{2}$ & 121.6 & 147.0 & 153.0 & 140.5 & 116.4 & 153.9 & 146.4 & 138.9 & 84.8 & 107.2 & 106.7 & 99.6 & 107.6 & 136.0 & 135.3 & 126.3 \\
\hline $\mathbf{F}_{3}$ & 190.7 & 244.8 & 151.4 & 195.6 & 125.8 & 193.1 & 158.4 & 159.1 & 87.7 & 110.8 & 110.0 & 102.8 & 134.7 & 182.9 & 139.9 & 152.5 \\
\hline Mean & 137.8 & 171.4 & 143.9 & 151.0 & 113.0 & 158.3 & 142.2 & 137.8 & 81.0 & 102.4 & 101.8 & 95.1 & 110.6 & 144.0 & 129.3 & 128.0 \\
\hline & $\mathbf{M}$ & I & $\mathbf{F}$ & M x I & $\mathbf{M} \times \mathbf{F}$ & I $\times \mathbf{F}$ & \multicolumn{10}{|c|}{$\mathbf{M} \times \mathbf{I} \times \mathbf{F}$} \\
\hline SE d & 0.844 & 0.955 & 1.079 & 1.593 & 1.744 & 1.800 & \multicolumn{10}{|c|}{2.969} \\
\hline $\begin{array}{c}\text { CD (0.05) } \\
\%\end{array}$ & 2.343 & 2.081 & 2.189 & 3.738 & 3.844 & 3.729 & \multicolumn{10}{|c|}{6.115} \\
\hline $\mathrm{CV} \%$ & \multicolumn{16}{|c|}{2.97} \\
\hline
\end{tabular}

Table.1b Influence of mulching, drip irrigation and fertigation on plant height $(\mathrm{cm})$ in nerium at flowering stage

\begin{tabular}{|c|c|c|c|c|c|c|c|c|c|c|c|c|c|c|c|c|}
\hline \multirow[t]{2}{*}{ Treatments } & \multicolumn{4}{|c|}{$\mathbf{M}_{1}$} & \multicolumn{4}{|c|}{$\mathbf{M}_{2}$} & \multicolumn{4}{|c|}{$\mathbf{M}_{3}$} & \multicolumn{4}{|c|}{ I $\mathbf{x} \mathbf{F}$} \\
\hline & $\mathbf{I}_{1}$ & $\mathbf{I}_{2}$ & $\mathbf{I}_{3}$ & Mean & $\mathbf{I}_{1}$ & $\mathbf{I}_{2}$ & $\mathbf{I}_{3}$ & Mean & $\mathbf{I}_{1}$ & $\mathbf{I}_{2}$ & $\mathbf{I}_{3}$ & Mean & $\mathbf{I}_{1}$ & $\mathbf{I}_{2}$ & $\mathbf{I}_{3}$ & Mean \\
\hline $\mathbf{F}_{1}$ & 157.8 & 203.4 & 190.8 & 184.0 & 150.9 & 199.6 & 194.5 & 181.7 & 110.0 & 136.8 & 132.1 & 126.3 & 139.5 & 179.9 & 172.4 & 157.8 \\
\hline $\mathbf{F}_{2}$ & 189.8 & 244.6 & 233.9 & 222.8 & 181.5 & 240.1 & 229.5 & 217.0 & 138.4 & 159.0 & 155.0 & 150.8 & 169.9 & 214.5 & 206.1 & 189.8 \\
\hline $\mathbf{F}_{3}$ & 196.3 & 277.0 & 255.3 & 242.9 & 187.7 & 270.3 & 265.9 & 241.3 & 166.4 & 268.0 & 262.0 & 232.1 & 183.4 & 271.7 & 261.0 & 196.3 \\
\hline Mean & 181.3 & 241.7 & 226.7 & 216.5 & 173.4 & 236.7 & 230.0 & 213.3 & 138.3 & 187.9 & 183.0 & 169.7 & 164.3 & 222.0 & 213.2 & 181.3 \\
\hline & M & I & $\mathbf{F}$ & M $\times I$ & $\mathbf{M} \times \mathbf{F}$ & $\mathbf{I} \times \mathbf{F}$ & \multicolumn{10}{|c|}{$\mathbf{M} \times \mathbf{I} \times \mathbf{F}$} \\
\hline SE d & 0.468 & 0.367 & 0.534 & 0.699 & 0.889 & 0.841 & \multicolumn{10}{|c|}{1.409} \\
\hline CD (0.05) \% & 1.300 & 0.800 & 1.085 & 1.711 & 1.988 & 1.730 & \multicolumn{10}{|c|}{2.888} \\
\hline CV \% & \multicolumn{16}{|c|}{2.52} \\
\hline
\end{tabular}


Table.2a Influence of mulching, drip irrigation and fertigation on number of primary branches plant ${ }^{-1}$ in nerium at pre flowering stage

\begin{tabular}{|c|c|c|c|c|c|c|c|c|c|c|c|c|c|c|c|c|}
\hline \multirow[t]{2}{*}{ Treatments } & \multicolumn{4}{|c|}{$\mathbf{M}_{1}$} & \multicolumn{4}{|c|}{$\mathbf{M}_{2}$} & \multicolumn{4}{|c|}{$\mathbf{M}_{3}$} & \multicolumn{4}{|c|}{$\mathbf{I} \times \mathbf{F}$} \\
\hline & $\mathbf{I}_{1}$ & $\mathbf{I}_{2}$ & $\mathbf{I}_{3}$ & Mean & $\mathbf{I}_{1}$ & $\mathbf{I}_{2}$ & $\mathbf{I}_{3}$ & Mean & $\mathbf{I}_{1}$ & $\mathbf{I}_{2}$ & $\mathbf{I}_{3}$ & Mean & $\mathbf{I}_{1}$ & $\mathbf{I}_{2}$ & $\mathbf{I}_{3}$ & Mean \\
\hline $\mathbf{F}_{1}$ & 5.01 & 5.11 & 5.33 & 5.15 & 4.98 & 5.21 & 5.45 & 5.21 & 4.15 & 4.61 & 4.47 & 4.41 & 4.71 & 4.97 & 5.08 & 4.92 \\
\hline $\mathbf{F}_{2}$ & 6.23 & 6.45 & 6.12 & 6.27 & 6.47 & 6.14 & 5.77 & 6.13 & 4.56 & 4.60 & 4.91 & 4.69 & 5.75 & 5.73 & 5.60 & 5.69 \\
\hline $\mathbf{F}_{3}$ & 6.32 & 7.03 & 6.54 & 6.63 & 6.25 & 6.98 & 6.51 & 6.58 & 4.41 & 4.94 & 4.91 & 4.75 & 5.68 & 6.31 & 5.98 & 5.99 \\
\hline Mean & 5.85 & 6.20 & 6.00 & 6.02 & 5.90 & 6.11 & 5.91 & 5.97 & 4.37 & 4.72 & 4.76 & 4.62 & 5.38 & 5.67 & 5.55 & 5.53 \\
\hline & $\mathbf{M}$ & I & $\mathbf{F}$ & M x I & $\mathbf{M} \times \mathbf{F}$ & $\mathbf{I} \times \mathbf{F}$ & \multicolumn{10}{|c|}{$\mathbf{M} \times \mathbf{I} \times \mathbf{F}$} \\
\hline SE d & 0.0595 & 0.032 & 0.040 & 0.075 & 0.082 & 0.066 & \multicolumn{10}{|c|}{0.109} \\
\hline CD $(0.05) \%$ & 0.165 & 0.070 & 0.082 & 0.192 & 0.200 & 0.136 & \multicolumn{10}{|c|}{0.225} \\
\hline CV \% & \multicolumn{16}{|c|}{2.71} \\
\hline
\end{tabular}

Table.2b Influence of mulching, drip irrigation and fertigation on number of primary branches plant ${ }^{-1}$ in nerium at flowering stage

\begin{tabular}{|c|c|c|c|c|c|c|c|c|c|c|c|c|c|c|c|c|}
\hline \multirow[t]{2}{*}{ Treatments } & \multicolumn{4}{|c|}{$\mathbf{M}_{1}$} & \multicolumn{4}{|c|}{$\mathbf{M}_{2}$} & \multicolumn{4}{|c|}{$\mathbf{M}_{3}$} & \multicolumn{4}{|c|}{ I $x \mathbf{F}$} \\
\hline & $\mathbf{I}_{1}$ & $\mathbf{I}_{2}$ & $\mathbf{I}_{3}$ & Mean & $\mathbf{I}_{1}$ & $\mathbf{I}_{2}$ & $\mathbf{I}_{3}$ & Mean & $\mathbf{I}_{1}$ & $\mathbf{I}_{2}$ & $\mathbf{I}_{3}$ & Mean & $\mathbf{I}_{1}$ & $\mathbf{I}_{2}$ & $\mathbf{I}_{3}$ & Mean \\
\hline $\mathbf{F}_{1}$ & 7.12 & 8.56 & 7.64 & 7.77 & 7.20 & 8.12 & 7.32 & 7.55 & 4.58 & 4.69 & 4.89 & 4.72 & 6.31 & 7.12 & 6.61 & 6.68 \\
\hline $\mathbf{F}_{2}$ & 8.15 & 8.45 & 8.21 & 8.27 & 7.23 & 7.83 & 8.41 & 7.82 & 4.72 & 5.11 & 4.92 & 4.92 & 6.70 & 7.13 & 7.18 & 7.00 \\
\hline $\mathbf{F}_{3}$ & 8.23 & 9.15 & 9.00 & 8.79 & 7.99 & 9.11 & 8.45 & 8.52 & 4.67 & 4.78 & 5.12 & 4.86 & 6.96 & 7.68 & 7.52 & 7.39 \\
\hline \multirow[t]{2}{*}{ Mean } & 7.83 & 8.72 & 8.28 & 8.28 & 7.47 & 8.35 & 8.06 & 7.96 & 4.66 & 4.86 & 4.98 & 4.83 & 6.66 & 7.31 & 7.10 & 7.02 \\
\hline & $\mathbf{M}$ & I & $\mathbf{F}$ & M $\mathbf{x} \mathbf{I}$ & $\mathbf{M} \times \mathbf{F}$ & I $\times \mathbf{F}$ & \multicolumn{10}{|c|}{$M \times I \times F$} \\
\hline SE d & 0.057 & 0.051 & 0.053 & 0.092 & 0.094 & 0.091 & \multicolumn{10}{|c|}{0.149} \\
\hline CD (0.05) \% & 0.159 & 0.112 & 0.107 & 0.223 & 0.217 & 0.189 & \multicolumn{10}{|c|}{0.307} \\
\hline CV \% & \multicolumn{16}{|c|}{2.78} \\
\hline
\end{tabular}


Table.3 Influence of mulching, drip irrigation and fertigation on days to first inflorescence emergence in nerium at flowering stage

\begin{tabular}{|c|c|c|c|c|c|c|c|c|c|c|c|c|c|c|c|c|}
\hline \multirow[t]{2}{*}{ Treatments } & \multicolumn{4}{|c|}{$\mathbf{M}_{1}$} & \multicolumn{4}{|c|}{$\mathbf{M}_{2}$} & \multicolumn{4}{|c|}{$\mathbf{M}_{3}$} & \multicolumn{4}{|c|}{$\mathbf{I} \times \mathbf{F}$} \\
\hline & $\mathbf{I}_{1}$ & $\mathbf{I}_{2}$ & $\mathbf{I}_{3}$ & Mean & $\mathbf{I}_{1}$ & $\mathbf{I}_{2}$ & $\mathbf{I}_{3}$ & Mean & $\mathbf{I}_{1}$ & $\mathbf{I}_{2}$ & $\mathbf{I}_{\mathbf{3}}$ & Mean & $\mathbf{I}_{1}$ & $\mathbf{I}_{2}$ & $\mathbf{I}_{3}$ & Mean \\
\hline $\mathbf{F}_{1}$ & 172.6 & 172.7 & 172.8 & 172.7 & 175.6 & 172.5 & 173.3 & 173.8 & 181.5 & 176.4 & 178.0 & 178.6 & 176.6 & 173.9 & 174.7 & 175.1 \\
\hline $\mathbf{F}_{2}$ & 172.3 & 172.0 & 172.4 & 172.2 & 175.4 & 172.1 & 173.0 & 173.5 & 180.2 & 177.4 & 177.7 & 178.4 & 176.0 & 173.8 & 174.4 & 174.7 \\
\hline $\mathbf{F}_{3}$ & 172.8 & 171.6 & 172.4 & 172.3 & 175.1 & 171.9 & 173.0 & 173.3 & 178.2 & 175.2 & 177.9 & 177.1 & 175.4 & 172.9 & 174.4 & 174.2 \\
\hline Mean & 172.6 & 172.1 & 172.5 & 172.4 & 175.4 & 172.2 & 173.1 & 173.5 & 180.0 & 176.3 & 177.9 & 178.1 & 176.0 & 173.5 & 174.5 & 174.7 \\
\hline & $\mathbf{M}$ & I & $\mathbf{F}$ & M x I & $\mathbf{M} \times \mathbf{F}$ & $\mathbf{I} \times \mathbf{F}$ & \multicolumn{10}{|c|}{$\mathbf{M} \mathbf{x} \mathbf{I} \mathbf{F}$} \\
\hline SE d & 1.407 & 1.067 & 1.415 & 2.063 & 2.446 & 2.268 & \multicolumn{10}{|c|}{3.781} \\
\hline CD $(0.05) \%$ & 3.907 & 2.325 & 2.870 & 5.069 & 5.568 & 4.676 & \multicolumn{10}{|c|}{7.760} \\
\hline CV \% & \multicolumn{16}{|c|}{2.84} \\
\hline
\end{tabular}

Table.4 Influence of mulching, drip irrigation and fertigation on days to first flower opening in nerium at flowering stage

\begin{tabular}{|c|c|c|c|c|c|c|c|c|c|c|c|c|c|c|c|c|}
\hline \multirow[t]{2}{*}{ Treatments } & \multicolumn{4}{|c|}{$\mathbf{M}_{1}$} & \multicolumn{4}{|c|}{$\mathbf{M}_{2}$} & \multicolumn{4}{|c|}{$\mathbf{M}_{3}$} & \multicolumn{4}{|c|}{ I $\times \mathbf{F}$} \\
\hline & $\mathbf{I}_{1}$ & $\mathbf{I}_{2}$ & $\mathbf{I}_{3}$ & Mean & $\mathbf{I}_{1}$ & $\mathbf{I}_{2}$ & $\mathbf{I}_{3}$ & Mean & $\mathbf{I}_{1}$ & $\mathbf{I}_{2}$ & $\mathbf{I}_{3}$ & Mean & $\mathbf{I}_{1}$ & $\mathbf{I}_{2}$ & $\mathbf{I}_{3}$ & Mean \\
\hline $\mathbf{F}_{1}$ & 180.49 & 178.64 & 179.56 & 179.56 & 184.78 & 183.00 & 183.98 & 183.92 & 185.90 & 183.92 & 184.99 & 184.94 & 183.72 & 181.85 & 182.84 & 182.80 \\
\hline $\mathbf{F}_{3}$ & 179.22 & 177.00 & 178.00 & 178.07 & 184.12 & 182.65 & 183.47 & 183.41 & 185.60 & 183.47 & 183.25 & 184.11 & 182.98 & 181.04 & 181.57 & 181.86 \\
\hline Mean & 179.75 & 178.02 & 178.63 & 178.80 & 184.52 & 182.81 & 183.70 & 183.67 & 185.78 & 183.75 & 184.17 & 184.57 & 183.35 & 181.52 & 182.16 & 182.35 \\
\hline & M & I & $\mathbf{F}$ & M x I & $\mathbf{M} \times \mathbf{F}$ & I $x$ F & \multicolumn{10}{|c|}{$\mathbf{M} \times \mathbf{I} \times \mathbf{F}$} \\
\hline SE d & 0.267 & 0.308 & 0.534 & 0.267 & 0.308 & 0.547 & \multicolumn{10}{|c|}{0.500} \\
\hline $\operatorname{CD}(0.05) \%$ & 0.554 & 0.640 & 1.108 & 0.554 & 0.640 & 0.557 & \multicolumn{10}{|c|}{1.112} \\
\hline CV \% & \multicolumn{16}{|c|}{2.65} \\
\hline
\end{tabular}


Table.5a Influence of mulching, drip irrigation and fertigation on cumulative flower yield $\mathrm{ha}^{-1}(\mathrm{~kg})$ in nerium from January 2017 to March 2017 at flowering stage

\begin{tabular}{|c|c|c|c|c|c|c|c|c|c|c|c|c|c|c|c|c|}
\hline \multirow[t]{2}{*}{ Treatments } & \multicolumn{4}{|c|}{$\mathbf{M}_{1}$} & \multicolumn{4}{|c|}{$\mathbf{M}_{2}$} & \multicolumn{4}{|c|}{$\mathbf{M}_{3}$} & \multicolumn{4}{|c|}{ I x F } \\
\hline & $\mathbf{I}_{1}$ & $\mathbf{I}_{2}$ & $\mathbf{I}_{3}$ & Mean & $\mathbf{I}_{1}$ & $\mathbf{I}_{2}$ & $\mathbf{I}_{3}$ & Mean & $\mathbf{I}_{1}$ & $\mathbf{I}_{2}$ & $\mathbf{I}_{3}$ & Mean & $\mathbf{I}_{1}$ & $\mathbf{I}_{2}$ & $\mathbf{I}_{3}$ & Mean \\
\hline $\mathbf{F}_{1}$ & 5425 & 6600 & 5300 & 5775 & 3975 & 5025 & 4250 & 4417 & 2100 & 2600 & 2325 & 2342 & 3833 & 4742 & 3958 & 4178 \\
\hline $\mathbf{F}_{2}$ & 5700 & 6975 & 5774 & 6150 & 4987 & 6200 & 5874 & 5687 & 2200 & 2750 & 2375 & 2442 & 4296 & 5308 & 4674 & 4759 \\
\hline $\mathbf{F}_{3}$ & 5925 & 13300 & 7800 & 9008 & 5550 & 8375 & 6350 & 6758 & 2245 & 3375 & 3150 & 2923 & 4573 & 8350 & 5767 & 6230 \\
\hline Mean & 5683 & 8958 & 6291 & 6978 & 4837 & 6533 & 5491 & 5621 & 2182 & 2908 & 2617 & 2569 & 4234 & 6133 & 4800 & 5056 \\
\hline & $\mathbf{M}$ & I & $\mathbf{F}$ & M x I & $\mathbf{M} \times \mathbf{F}$ & $\mathbf{I} \times \mathbf{F}$ & \multicolumn{10}{|c|}{$\mathbf{M} \times \mathbf{I} \times \mathbf{F}$} \\
\hline SE d & 47.37 & 37.61 & 36.63 & 71.23 & 70.20 & 64.01 & \multicolumn{10}{|c|}{104.31} \\
\hline CD $(0.05) \%$ & 131.5 & 81.95 & 74.30 & 174.03 & 166.30 & 133.17 & \multicolumn{10}{|c|}{215.65} \\
\hline CV \% & \multicolumn{16}{|c|}{2.85} \\
\hline
\end{tabular}

Table.5b Influence of mulching, drip irrigation and fertigation on cumulative flower yield ha ${ }^{-1}(\mathrm{~kg})$ in nerium from April 2017 to August 2017 at peak flowering stage

\begin{tabular}{|c|c|c|c|c|c|c|c|c|c|c|c|c|c|c|c|c|}
\hline \multirow[t]{2}{*}{ Treatments } & \multicolumn{4}{|c|}{$\mathbf{M}_{1}$} & \multicolumn{4}{|c|}{$\mathbf{M}_{2}$} & \multicolumn{4}{|c|}{$\mathbf{M}_{3}$} & \multicolumn{4}{|c|}{ I x F } \\
\hline & $\mathbf{I}_{1}$ & $\mathbf{I}_{2}$ & $\mathbf{I}_{3}$ & Mean & $\mathbf{I}_{1}$ & $\mathbf{I}_{2}$ & $\mathbf{I}_{3}$ & Mean & $\mathbf{I}_{1}$ & $\mathbf{I}_{2}$ & $\mathbf{I}_{3}$ & Mean & $\mathbf{I}_{1}$ & $\mathbf{I}_{2}$ & $\mathbf{I}_{3}$ & Mean \\
\hline $\mathbf{F}_{1}$ & 7524 & 9150 & 7625 & 8100 & 5247 & 5775 & 5552 & 5525 & 3350 & 4875 & 4756 & 4327 & 5374 & 6600 & 5978 & 5984 \\
\hline $\mathbf{F}_{2}$ & 7300 & 10050 & 8225 & 8525 & 5364 & 6457 & 5647 & 5823 & 3650 & 4987 & 4675 & 4437 & 5438 & 7165 & 6182 & 6262 \\
\hline $\mathbf{F}_{3}$ & 6700 & 18300 & 11875 & 12292 & 5478 & 15850 & 6500 & 9276 & 4575 & 5124 & 5001 & 4900 & 5584 & 13091 & 7792 & 8822 \\
\hline Mean & 7175 & 12500 & 9242 & 9639 & 5363 & 9361 & 5900 & 6874 & 3858 & 4995 & 4811 & 4555 & 5465 & 8952 & 6651 & 7023 \\
\hline & $\mathbf{M}$ & I & $\mathbf{F}$ & M x I & $\mathbf{M} \times \mathbf{F}$ & $\mathbf{I} \times \mathbf{F}$ & \multicolumn{10}{|c|}{$M \times I \times F$} \\
\hline SE d & 58.68 & 55.49 & 53.95 & 97.99 & 96.26 & 94.34 & \multicolumn{10}{|c|}{153.7} \\
\hline CD (0.05) \% & 162.9 & 120.9 & 109.4 & 234.5 & 222.0 & 196.2 & \multicolumn{10}{|c|}{317.7} \\
\hline CV \% & \multicolumn{16}{|c|}{2.86} \\
\hline
\end{tabular}


Table.5c Influence of mulching, drip irrigation and fertigation on cumulative flower yield $\mathrm{ha}^{-1}(\mathrm{~kg})$ in nerium from September 2016 to October2017 at lean flowering stage

\begin{tabular}{|c|c|c|c|c|c|c|c|c|c|c|c|c|c|c|c|c|}
\hline \multirow[t]{2}{*}{ Treatments } & \multicolumn{4}{|c|}{$\mathbf{M}_{1}$} & \multicolumn{4}{|c|}{$\mathbf{M}_{2}$} & \multicolumn{4}{|c|}{$\mathbf{M}_{3}$} & \multicolumn{4}{|c|}{$\mathbf{I} \times \mathbf{F}$} \\
\hline & $\mathbf{I}_{1}$ & $\mathbf{I}_{2}$ & $\mathbf{I}_{3}$ & Mean & $\mathbf{I}_{1}$ & $\mathbf{I}_{2}$ & $\mathbf{I}_{3}$ & Mean & $\mathbf{I}_{1}$ & $\mathbf{I}_{2}$ & $\mathbf{I}_{3}$ & Mean & $\mathbf{I}_{1}$ & $\mathbf{I}_{2}$ & $\mathbf{I}_{3}$ & Mean \\
\hline $\mathbf{F}_{1}$ & 5025 & 6400 & 5825 & 5750 & 3200 & 5825 & 3800 & 4275 & 975 & 2475 & 1650 & 1700 & 3067 & 4900 & 3758 & 3908 \\
\hline $\mathbf{F}_{2}$ & 5325 & 6525 & 6000 & 5950 & 3375 & 6175 & 5426 & 4992 & 1075 & 2575 & 1975 & 1875 & 3258 & 5092 & 4467 & 4272 \\
\hline $\mathbf{F}_{3}$ & 5525 & 7625 & 7100 & 6750 & 4001 & 7450 & 6965 & 6139 & 1375 & 2875 & 2725 & 2325 & 3634 & 5983 & 5597 & 5071 \\
\hline Mean & 5292 & 6850 & 6308 & 6150 & 3525 & 6483 & 5397 & 5135 & 1142 & 2642 & 2117 & 1967 & 3320 & 5325 & 4607 & 4417 \\
\hline & M & I & $\mathbf{F}$ & $\mathbf{M} \times \mathbf{I}$ & $\mathbf{M} \times \mathbf{F}$ & $\mathbf{I} \times \mathbf{F}$ & \multicolumn{10}{|c|}{$M \times I \times F$} \\
\hline SE d & 27.59 & 24.83 & 27.90 & 44.66 & 48.15 & 46.62 & \multicolumn{10}{|c|}{76.85} \\
\hline CD (0.05) \% & 76.62 & 54.11 & 56.60 & 107.5 & 109.5 & 96.57 & \multicolumn{10}{|c|}{158.2} \\
\hline CV \% & \multicolumn{16}{|c|}{2.39} \\
\hline
\end{tabular}


The treatment $\mathrm{I}_{2} \mathrm{~F}_{3}(100 \%$ WRc through drip irrigation $+125 \% \mathrm{RDF}$ through fertigation) recorded the least days to first inflorescence emergence (172.9 days) and days to first flower opening(181.04 days respectively). The three way interaction effects of mulching, drip irrigation and fertigation were significant with respect to days taken to first inflorescence emergence and days to first flower opening. The treatment $\mathrm{M}_{1} \mathrm{I}_{2} \mathrm{~F}_{3}$ (black polythene mulch $+100 \%$ WRc through drip irrigation $+125 \%$ RDF through fertigation) recorded the least days to first inflorescence emergence and days to first flower opening (171.6 and 181.04 days) which was on par with treatments $\mathrm{M}_{2} \mathrm{I}_{2} \mathrm{~F}_{3}$. Higher levels of recommended dose of fertilizers reduced the time taken for flower initiation as compared to 100 per cent recommended dose of fertilizers. Early flower initiation may be due to the increased levels of potassium given to the plants in the water soluble form, better utilization of soil moisture, soil nutrients, less weed intensity throughout crop growth stage which might have enhanced the reproductive development of the plant (Yathindra, 2009) in china aster.

Similar results were reported by Chaitra (2006) in china aster and Ashutosh Sharma (2013) in tuberose. Early flower initiation may be due to the fact that plants got sufficient moisture for longer time and maintained turgidity resulting in better uptake of nutrients and lower weed intensity. Similar findings were reported by Vijay Kumar (2009) in China aster, Vasanthakumari et al., 2013 in gladiolus; Alak Barman et al., (2015) in tuberose; Adnan Younis et al., (2012) in freesia; Pal and Ghosh (2010) and Iftikhar Ahmad et al., (2011) in african marigold.

With regard to main plots, the treatment $M_{1}$ (black polythene mulch) registered higher flower yield of 6978, 9639 and $6150(\mathrm{~kg}) \mathrm{ha}^{-1}$ from January 2017 to March 2017, April 2017 to August 2017 and September 2017 to October 2017 respectively (Table 5 a, 5 b and $5 \mathrm{c})$. Among the sub plot treatments, $\mathrm{I}_{2}$ (100 $\%$ WRc through drip irrigation) registered the higher flower yield of 6133, 8952 and 5325 kg from January 2017 to March 2017, April 2017 to August 2017 and September 2017 to October 2017 respectively.

Among the three sub plot fertigation treatments, $\mathrm{F}_{3} \quad(125 \%$ RDF through fertigation) registered the higher flower yield of 6230, 8822 and $5071 \mathrm{~kg}$ from January 2017 to March 2017, April 2017 to August 2017 and September 2017 to October 2017 respectively.

Among the interaction effects the treatment combination $\mathrm{M}_{1} \mathrm{I}_{2}$ (Black polythene mulch + $100 \%$ WRc through drip irrigation) had recorded higher flower yield of 8958, 12500 and $6850 \mathrm{~kg}$ from January 2017 to March 2017, April 2017 to August 2017 and September 2017 to October 2017 respectively.

The interaction effect between mulching and fertigation significantly influenced flower yield. The treatment, $\mathrm{M}_{1} \mathrm{~F}_{3}$ (black polythene mulch $+125 \%$ RDF through fertigation) recorded higher flower yield (9008, 12292 and $6750 \mathrm{~kg}$ ) from January 2017 to March 2017, April 2017 to August 2017 and September 2017 to October 2017 respectively. The interaction effects between drip irrigation and fertigation indicated that the treatment, $\mathrm{I}_{2} \mathrm{~F}_{3}(100 \%$ WRc through drip irrigation $+125 \% \mathrm{RDF}$ through fertigation) recorded the highest flower yield (8350, 13091 and $5983 \mathrm{~kg}$ ) from January 2017 to March 2017, April 2017 to August 2017 and September 2017 to October 2017.

The combined effect of application of black polythene mulch $+100 \%$ WRc through drip irrigation $+125 \%$ RDF through fertigation 
$\left(\mathrm{M}_{1} \mathrm{I}_{2} \mathrm{~F}_{3}\right)$ registered higher flower yield from January 2017 to March 2017, April 2017 to August 2017 and September 2017 to October $2017(13300,18300$ and $7625 \mathrm{~kg})$ and this was on par with $\mathrm{M}_{2} \mathrm{I}_{2} \mathrm{~F}_{3}$ - coir waste $+100 \%$ WRc through drip irrigation $+125 \% \mathrm{RDF}$ through fertigation $(8375,15850$ and 7450 $\mathrm{kg}$ ). The results obtained in this study demonstrate that the effects of quality of irrigation water and irrigation frequency are significantly important in order to obtain higher yield. This is in line with the findings by Koksal Aydinsakir et al., (2011) in carnation, Bagali et al., (2012) in onions; Lodhi et al., (2014) in sweet pepper; ArifTuran et al., (2015) in cut chrysanthemum; Ayyanna et al., (2014) in marigold; Puneet Sharma and Arun Kaushal (2015); Ughade and Mahadkar (2015) in brinjal and Muthu Kumar and Ponnuswami (2013) in noni. Nutrients like N, P and K are being the constituent of proteins, amino acids, nucleic acid, various enzymes and coenzymes is associated with the increased shoot length and leaf area resulting in more photosynthesis and thus increased transformation of manufactured food material from source (leaf) to sink (flower bud) as reported by Vinoth (2012) in lilium hybrids; Khalid Elhindi et al., (2015) in Zinnia and Jakadeeshkanth, (2014) in curry leaf. The present findings are in agreement with the findings of Rafat Saeed and Rafiq Ahmad, (2009) in tomato and Tomasz Spizewski et al., (2010) in cucumber

\section{References}

Ayyanna D. Siddapur., Basavaraj, S. Polisgowdar, Rajan and Hiremath, Muddi Nemichandrappa, Satish Kumar, Ashok H. Hugar, Shivan and N. Honnali and Gurappa S. Yadahalli. 2014. Evaluation of surface and drip irrigation methods for marigold flower (Tagetes erecta L) under Raichur condition.

Adnan Younis, Muhammad Zahid Mukhtar Bhatti, Atif Riaz, Usman Tariq,
Muhammad Arfan, Muhammad Nadeem and Muhammad Ahsan. 2012. Effect of different types of mulching on growth and flowering of Freesia alba cv. Aurora. Pak. J. Agri. Sci., 49(4):429-433.

Alak Barman, Abu Habib M.D. Abdullah, Arman Hossen, M.D. Asrafuzzaman and M. Habibur Rahman. 2015. Effect of different mulching on growth and yield of tuberose. International J of Res \& Review., 2(6): 301.

ArifTuran, Yusuf Ucar and Soner Kazaz. 2015. Effects of different irrigation treatment on quality parameters of cut chrysanthemum. Scientific Papers. Series B, Horticulture. LIX,

Ashutosh Sharma. 2013. Effect of nitrogen and phosphorous on growth and flowering in tuberose (Polianthes tuberosa L.) cv. Double. MSc., (Hort) Thesis, Y.S.R. Horticultural University, Solan.

Bagali, A.N., H.B. Patil, M.B. Guled and R.V. Patil. 2012. Effect of scheduling of drip irrigation on growth, yield and water use efficiency of onion (Allium cepaL.). Karnataka J. Agric. Sci., 25 (1): (116-119).

Chaitra R. 2006. Effect of integrated nutrient management on growth, yield and quality of china aster (Callistephus chinensis (L.) Nees). University of Agricultural Sciences, Dharwad.

Chawla, S. L. 2008. Response of african marigold to irrigation and mulching. J. Ornt. Hort., 11(2):131-135.

Iftikhar Ahmad, Muhammad Asif, AtyabAmjad, Sagheer Ahmad. 2011. Fertilization enhances growth, yield, and xanthophyll contents of marigold. Turk J Agric For 35: 641-648.

Jainag, K., K. V. Jayaprasad, R. Krishnamanohar, Shivan and Hongal and K. Prakash. 2011. Effect of levels of fertigation on growth and yield of bird of paradise (Strelitzia reginae Ait). Asian J. Hort., 6(1):118-121.

Jakadeeshkanth, R. 2014. Standardization of fertigation scheduling and high density planting system in curry leaf (Murraya koenigii Spreng) for high yield and quality. Ph.D Thesis, Tamil Nadu Agricultural University., Periyakulam.

Khalid Elhindi, Salah El Hendawy, Eslam Abdel 
Salam, Abdallah Elgorban, Mukhtar Ahmed. 2015. Impacts of fertigation via surface and subsurface drip irrigation on growth rate, yield and flower quality of Zinnia elegans, Bragantia, Campinas., 20(10): 1-12.

Koksal Aydinsakir, Ismail HakkiTuzel and Dursun Buyuktas. 2011. The effects of different irrigation levels on flowering and flower quality of carnation (Dianthus caryophllus L.) irrigated by drip irrigation. African J of Biotechnol 10 (66):1482614835.

Lodhi, A. S., A. Kaushal and K.G. Singh. 2014. Impact of irrigation regimes on growth, yield and water use efficiency of sweet pepper. Indian Journal of Science and Technology, 7(6), 790-794.

Muthu Kumar, S. and V. Ponnuswami. 2013. Effect of different water regimes and organic manures on quality parameters of noni (Morinda citrifolia).8(27) 3534-3543.

Pal, P. and P Ghosh. 2010. Effect of different sources and levels of potassium on growth flowering and yield of african marigold $\mathrm{cv}$. Siracole. Indian $\mathbf{J}$ of natural products and resources. 1 (3):371-375.

Panse, V.G. and P.V. Sukhatme. 1985. Statistical methods for agricultural works. Fourth Edn., ICAR, New Delhi.

Patel Bhavesh kumar Bharatbhai. 2013. Effect of irrigation levels and mulching on growth and yield of tuberose (Polianthes tuberosa L.) var. Prajwal. M.Sc (Hort) Thesis, Navsari Agricultural University.

Puneet Sharma and Arun Kaushal. 2015. Growing okra with drip irrigation and fertigation a review. International J of Engineering Sci. Invention. 4 (9):01-05.

Rafat Saeed and Rafiq Ahmad. 2009. Vegetative growth and yield of tomato as affected by the application of organic mulch and gypsum under saline rhizosphere Pak. J. Bot., 41(6): 3093-3105.

Tomasz Spizewski, Barbara Frąszczak, Alina Kałużewicz, Włodzimierz Krzesinski and Jolanta Lisiecka. 2010. The effect of black polyethylene mulch on yield of field grown cucumber. Acta Sci. Pol., Hortorum Cultus 9(3): 221-229.

Ughade, S. R. and U. V. Mahadkar. 2015. Effect of different planting density, irrigation and fertigation levels on growth and yield of brinjal (Solanum melongena L.). The bio sci, 10 (3): 1205-1211.

Vasanthakumari. R., D.P. Kumar , B. Arun Kumar and M. Mahadevamma (2013). Effect of plant density, planting methods and mulching on floral and cormal parameters in gladiolus (Gladiolus hybridus L.). The Asian J. horti., 8(3): 391-398.

Vijaya Kumar, N. S. 2009. Standardisation of drip irrigation and fertigation in china aster (Callistephus chinensis L. Nees). M.Sc (Hort) Thesis, University of Agricultural Sciences, Bangalore.

Vinoth. 2012. Studies on optimization of fertigation schedule for improved growth, yield and quality of Asiatic and OT hybrid Lilies (Lilium spp.). Ph.D Thesis, Tamil Nadu Agricultural University., Coimbatore.

Yathindra, H.A. 2009. Effect of plastic mulching and fertigation on growth, yield and flower quality of china aster (Callistephus chinensis L. Nees). M.Sc (Hort) Thesis, University of Agricultural Sciences, Bangalore.

\section{How to cite this article:}

Annasamy, K., S. Muthu Lakshmi, K. M. Sellamuthu, T. Thangaselvabai, J. Kannan, T. L. Preethi and Arularasu, P. 2020. Effect of Mulching, Drip irrigation and Fertigation on Growth, Flowering and Yield parameters of Nerium (Nerium oleander L.). Int.J.Curr.Microbiol.App.Sci. 9(11): 2272-2282. doi: https://doi.org/10.20546/ijcmas.2020.911.272 